University of Wollongong

Research Online

Faculty of Engineering - Papers (Archive)

Faculty of Engineering and Information

Sciences

2009

\title{
Voltage support by distributed generation units and shunt capacitors in distribution systems
}

\author{
Kai Zou \\ University of Wollongong \\ Ashish Agalgaonkar \\ University of Wollongong, ashish@uow.edu.au \\ Kashem M. Muttaqi \\ University of Wollongong, kashem@uow.edu.au \\ Sarath Perera \\ University of Wollongong, sarath@uow.edu.au
}

Follow this and additional works at: https://ro.uow.edu.au/engpapers

Part of the Engineering Commons

https://ro.uow.edu.au/engpapers/5473

\section{Recommended Citation}

Zou, Kai; Agalgaonkar, Ashish; Muttaqi, Kashem M.; and Perera, Sarath: Voltage support by distributed generation units and shunt capacitors in distribution systems 2009.

https://ro.uow.edu.au/engpapers/5473

Research Online is the open access institutional repository for the University of Wollongong. For further information contact the UOW Library: research-pubs@uow.edu.au 


\title{
Voltage Support by Distributed Generation Units and Shunt Capacitors in Distribution Systems
}

\author{
Kai Zou, A. P. Agalgaonkar, K. M. Muttaqi, Senior Member, IEEE, and S. Perera, Member, IEEE
}

\begin{abstract}
Integration of distributed generation (DG) units and shunt capacitors in the radial distribution networks is one of the effective options that can be used to improve the system voltage and reduce system losses. Optimal sizing and siting of DG units and shunt capacitors need to be ensured for strengthening the supply quality and reliability of distribution systems. In this regard, new analytical strategies need to be devised to minimise the computational burden and improve the overall accuracy of the solution. In this paper, a numerical method for the identification of the target voltage support zones is proposed by reducing the large search space. The strategic placement of DG units and shunt capacitors is proposed for overall voltage support and power loss reduction in a distribution feeder. The investment cost for DG units and shunt capacitors is minimised by using particle swarm optimisation (PSO) technique.
\end{abstract}

Index Terms-Distributed generation, shunt capacitor, voltage support, power loss, optimisation.

\section{INTRODUCTION}

With the developments in DG technologies, their integration for distribution system upgrades is an attractive alternative planning option for distribution utilities [1]. DGs can support system voltage, minimise system losses and alleviate the distribution system expansion costs. On the other hand, the installation of shunt capacitors is another cost-effective solution to improve system voltage and minimise losses [2].

In order to maximise the benefits for the integration of DG units and shunt capacitors, their sizes and locations need to be identified using systematic approaches. The optimal sizing and siting of DG units and shunt capacitors in distribution systems is a complex optimisation problem. This combinatorial problem can be solved by either conventional mathematical programming methods such as sequential quadratic programming (SQP), branch and bound method or other heuristic techniques such as genetic algorithm (GA), ant colony optimisation (ACO), particle swarm optimisation(PSO). In [3], Grainger and Lee have proposed an analytical approach to obtain the optimum sizes and locations of shunt capacitors for loss minimisation. In [4]-[6], heuristic search methods based on the analysis of sensitivity indices have been proposed for minimising the cost investment with shunt capacitors. In [7][10], intelligent-based heuristic methods have been justified for distribution system planning.

This work is supported by Integral Energy, New South Wales, Australia

Kai Zou, A. P. Agalgaonkar, K. M. Muttaqi and S. Perera are with the Integral Energy Power Quality and Reliability Centre, School of Electrical, Computer, and Telecommunications Engineering, University of Wollongong, Wollongong, New South Wales, 2522, Australia. (e-mail: kz965@uow.edu.au; ashish@uow.edu.au; kashem@uow.edu.au; sarath@uow.edu.au)

978-1-4244-4241-6/09/\$25.00 C2009 IEEE
In this paper, the voltage support zones are identified for the radial distribution network by using an analytical approach based on the manipulation of system Jacobian matrix. The maximum voltage support is ensured with minimum real and reactive power injection at selective system nodes and minimum financial investment. The use of identified zones can avoid exhaustive search for all system nodes. Thus, the computational burden associated with the optimisation algorithms can be reduced; thereby improving the overall efficiency of the solutions. The proposed approach could be useful for sizing and siting of DG units and shunt capacitors in the radial distribution systems.

This paper is structured as follows: The proposed analytical approach for zone identification is developed in Section II. In order to compare the relationship between voltage improvement and loss reduction (for the same active and reactive power injection), the technique for maximum loss reduction is introduced in Section III. The cost optimisation for maximum voltage support with the integration of DG units and shunt capacitors is formulated in Section IV. The identified voltage support zones and corresponding loss reduction, together with the optimisation results are analysed and discussed in Section $\mathrm{V}$.

\section{ZONE IDENTIFICATION FOR VOLTAGE SUPPORT}

The siting and sizing of DG units and shunt capacitors for radial distribution networks should be strategically decided in order to have effective voltage improvement at all the nodes with minimum financial investment. In order to reduce the overall search space and improve the accuracy of the solution, selective number of nodes (zone) with the possibility of optimal solution need to be identified.

The voltage support zones with the selective number of nodes are identified for efficient real $(\mathrm{P})$ and reactive $(\mathrm{Q})$ injection by means of DG units (operated with unity power factor) and shunt capacitors. In the following subsections, the reduced Jacobian matrix is first derived using matrix manipulation and subsequently, the identification of voltage support zones for radial distribution networks is presented. Finally, the proposed approach is summarised.

\section{A. Reduced Jacobian Matrix}

The Jacobian matrix at different loading levels can be obtained by using Newton-Raphson power flow formulation and the relationships between the incremental change of $P$ and $Q$ injections and the incremental change of voltage angle and voltage magnitude can be established as [11]: 


$$
\left[\begin{array}{c}
\Delta P \\
\Delta Q
\end{array}\right]=\left[\begin{array}{ll}
J_{p \delta} & J_{p v} \\
J_{q \delta} & J_{q v}
\end{array}\right]\left[\begin{array}{c}
\Delta \delta \\
\Delta|V|
\end{array}\right]
$$

where $\Delta P$ and $\Delta Q$ are the column vectors, which are the incremental changes of $P$ and $Q$ at each $P Q$ node respectively, $\Delta \delta$ is the column vector for incremental change of voltage $\delta$ at each $P Q$ node, $\Delta|V|$ is the column vector for incremental change of voltage magnitude $|V|$ at each $P Q$ node, $J_{p \delta}$ and $J_{p v}$ represent the linearised relationships between $\Delta P$ and $\Delta \delta$ and $\Delta|V|$ respectively, $J_{q \delta}$ and $J_{q v}$ represent the linearised relationships between $\Delta Q$ and $\Delta \delta$ and $\Delta|V|$ respectively.

From (1), it is known that the magnitudes of system nodal voltages are affected by the incremental change of both $P$ and $Q$ injections. However, if one of the control variables can be kept constant, the improvement of system voltage profile can be estimated by considering the incremental change of another control variable [11]. In order to obtain a direct relationship between incremental change of voltage magnitude $\Delta|V|$ with the incremental real power injection $\Delta P$, it is assumed that the incremental reactive power injection $\Delta Q=0$. Hence (1) can be reduced as:

$$
\Delta P=\left[J_{p v}-J_{p \delta} J_{q \delta}^{-1} J_{q v}\right] \Delta|V|=J_{R} \Delta|V|
$$

Let $J_{R^{\prime}}=J_{R}^{-1}$, thus (2) can be rewritten as:

$$
\Delta|V|=J_{R^{\prime}} \Delta P
$$

Similarly, for $\Delta P=0$ :

$$
\begin{aligned}
\Delta Q & =\left[J_{q v}-J_{q \delta} J_{p \delta}^{-1} J_{p v}\right] \Delta|V|=J_{X} \Delta|V| \\
\Delta|V| & =J_{X^{\prime}} \Delta Q
\end{aligned}
$$

It can be observed that the element $J_{R^{\prime}(i, j)}$ in the matrix $J_{R^{\prime}}$ is the relationship between the incremental real power injection $\Delta P$ at node $j$ and the incremental voltage change $\Delta|V|$ at node $i$ with only incremental real power injections. Similarly, the element $J_{X^{\prime}(i, j)}$ in matrix $J_{X^{\prime}}$ is the relationship between the incremental reactive power injection $\Delta Q$ at node $j$ and the incremental voltage change $\Delta|V|$ at node $i$ with only incremental reactive power injections.

It should be noted that the linearised relationships given by (3) and (5) are only valid at a particular operating point with small incremental $\Delta P$ or $\Delta Q$ injections. The relationships between power injections and voltage magnitudes will dynamically change due to the inherent nonlinear $P-V$ and $Q-V$ characteristics. However, the correction to these relationships can be made by integrating the predicted incremental $\Delta P$ or $\Delta Q$ injection into the system and performing the power flow calculations iteratively. Initially, the predicted incremental $\Delta P$ or $\Delta Q$ injections can be obtained based on (3) and (5) with the assumption of small incremental real or reactive power injection and acceptable voltage limits. The detailed derivation of predicted indices and the correction procedure for the same indices will be described in the next subsection.

\section{B. Identification of Voltage Support Zones}

In this subsection, the reduced Jacobian matrices $J_{R^{\prime}}$ and $J_{X^{\prime}}$ are used to identify the independent target voltage support zones with DG units or shunt capacitors. It is assumed that the change in the nodal voltage $\Delta|V|$ is relatively small with the real and reactive power injections, consequently the linearised relationship matrix $J_{R^{\prime}}$ and $J_{X^{\prime}}$ will remain unchanged. The approach for identifying the voltage support zone with $P$ injection for radial distribution systems is developed in this subsection. The same approach can be used for $Q$ injection.

From (3), it can be observed that the changes in nodal voltages due to incremental $\Delta P$ injection at node $k$ can be expressed as:

$$
\left[\begin{array}{c}
\Delta\left|V_{1}\right| \\
\vdots \\
\Delta\left|V_{k}\right| \\
\vdots \\
\Delta\left|V_{n}\right|
\end{array}\right]=\left[\begin{array}{ccccc}
J_{R^{\prime}(11)} & \cdots & J_{R^{\prime}(1 k)} & \cdots & J_{R^{\prime}(1 n)} \\
\vdots & \ddots & \vdots & \ddots & \vdots \\
J_{R^{\prime}(k 1)} & \cdots & J_{R^{\prime}(k k)} & \cdots & J_{R^{\prime}(k n)} \\
\vdots & \ddots & \vdots & \ddots & \vdots \\
J_{R^{\prime}(n 1)} & \cdots & J_{R^{\prime}(n k)} & \cdots & J_{R^{\prime}(n n)}
\end{array}\right]\left[\begin{array}{c}
0 \\
\vdots \\
\Delta P_{k} \\
\vdots \\
0
\end{array}\right]
$$

The voltage improvement at each node due to incremental $\Delta P$ injection at node $k$ can be rewritten as:

$$
\left[\begin{array}{c}
\Delta\left|V_{1}\right| \\
\vdots \\
\Delta\left|V_{k}\right| \\
\vdots \\
\Delta\left|V_{n}\right|
\end{array}\right]=\left[\begin{array}{c}
J_{R^{\prime}(1 k)} \\
\vdots \\
J_{R^{\prime}(k k)} \\
\vdots \\
J_{R^{\prime}(n k)}
\end{array}\right] \Delta P_{k}
$$

It can be seen from (7) that the incremental $\Delta P$ injection at node $k$ can result in voltage improvement at all system nodes. The improvement of the system nodal voltages depends on the corresponding $k t h$ column of $J_{R^{\prime}}$ matrix and the amount of incremental $\Delta P$ injection. If the desired voltage improvement at each system node is known, the corresponding incremental $\Delta P$ injection at a specified location can be calculated.

In order to calculate the desired voltage improvement, it is assumed that the acceptable lower voltage limit for the system is $V_{\text {low }}$. The magnitude of nodal voltages $\left|V_{\text {node }}\right|$ can be obtained from the load flow calculation. The desired improvement of voltages $\Delta V_{d}$ between the minimum acceptable voltage $V_{\text {low }}$ and the nodal voltages $\left|V_{\text {node }}\right|$ at each node can be calculated as:

$$
\Delta V_{d}=V_{\text {low }}-\left|V_{\text {node }}\right|
$$

Since desired voltage improvement $\Delta V_{d}$ at each node is specified, then the corresponding desired incremental $\Delta P$ injection at node $k$ can be obtained as:

$$
\left[\begin{array}{c}
\Delta P_{1 k} \\
\vdots \\
\Delta P_{k k} \\
\vdots \\
\Delta P_{n k}
\end{array}\right]=\left[\begin{array}{c}
J_{R^{\prime}(1 k)}^{-1} \Delta V_{d(1)} \\
\vdots \\
J_{R^{\prime}(k k)}^{-1} \Delta V_{d(k)} \\
\vdots \\
J_{R^{\prime}(n k)}^{-1} \Delta V_{d(n)}
\end{array}\right]
$$

In (9), the matrix on the left hand side is the different amounts of the desired incremental $\Delta P$ injections at node $k$ 
in order to satisfy the different desired voltage improvement at each system node. Thus, by applying (9) at each system node independently, the desired incremental $\Delta P$ injections can be expressed in a general matrix form:

$$
P_{d}=\left[\begin{array}{ccc}
\Delta P_{d(11)} & \cdots & \Delta P_{d(1 n)} \\
\vdots & \ddots & \vdots \\
\Delta P_{d(n 1)} & \cdots & \Delta P_{d(n n)}
\end{array}\right]
$$

It can be seen that, for instance, the element $\Delta P_{d(1 n)}$ in the matrix $P_{d}$ is the desired incremental $\Delta P$ injection at node $n$ to maintain the voltage of node 1 in specified limits. The $P_{d}$ can be used in conjunction with the feeder topology matrix to derive $P_{d}^{u p}$ and $P_{d}^{l o}$. The $P_{d}^{u p}$ is an $n \times n$ matrix indicating the minimum desired incremental $\Delta P$ injection to keep the voltages at all the upstream nodes (with respect to the injection node) in specified limits while $P_{d}^{l o}$ is an $n \times n$ matrix to maintain the voltages at downstream nodes. The elements in $P_{d}^{u p}$ and $P_{d}^{l o}$ can be derived as:

$$
\begin{gathered}
P_{d(i j)}^{u p}=P_{d(i j)} M_{u p(i j)} \\
P_{d(i j)}^{l o}=P_{d(i j)} M_{l o(i j)}
\end{gathered}
$$

where $M_{u p}$ is an $n \times n$ binary matrix indicating the upstream nodes with respect to the corresponding injection nodes (eg. $M_{u p(i j)}=1$ indicates that node $i$ is located on the upstream side of node $j$ ) and $M_{l o}$ is an $n \times n$ binary matrix indicating the downstream nodes with respect to the corresponding injection nodes.

The maximum values in the each column of $P_{d}^{u p}$ are the minimum incremental $\Delta P$ injections for maintaining the voltages at all upstream nodes. Similarly, the maximum values in each column of $P_{d}^{l o}$ is the minimum incremental $\Delta P$ injections for maintaining the voltages at all downstream nodes. Thus,

$$
\begin{aligned}
& P_{\min (i)}^{u p}=\max \left(P_{d(i)}^{u p}\right) \\
& P_{\min (i)}^{l o}=\max \left(P_{d(i)}^{l o}\right)
\end{aligned}
$$

where $P_{d(i)}^{u p}$ is the $i$ th column of $P_{d}^{u p}, P_{d(i)}^{l o}$ is the $i t h$ column of $P_{d}^{l o}$.

The larger value of $P_{\min (i)}^{u p}$ and $P_{m i n(i)}^{l o}$ for node $i$ is the minimum amount of incremental $\Delta P$ injection $\left(P_{\min (i)}\right)$, which can keep all nodal voltages within specified limits. Thus,

$$
P_{\min (i)}= \begin{cases}P_{\min (i)}^{u p} & \text { if } P_{\min (i)}^{u p}>P_{\min (i)}^{l o} \\ P_{\min (i)}^{l o} & \text { otherwise }\end{cases}
$$

In some cases, the $P_{\min (i)}$ at node $i$ may exceed the maximum allowable incremental $\Delta P$ injection at that node. In such a case, the maximum allowable incremental $\Delta P$ injection $\left(P_{a(i)}^{\max }\right)$ should be identified and the voltage support zone should be expanded to allow multiple $P$ injections.

The $P_{a(i)}^{\max }$ are determined by the upper voltage limit $V_{u p}$ and the injection location. The allowable voltage rise at each node can be calculated as:

$$
\Delta V_{a}=V_{\text {up }}-\left|V_{\text {node }}\right|
$$

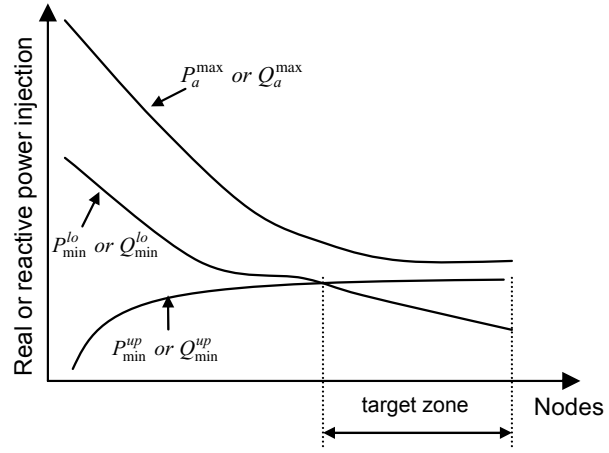

Fig. 1. Identification of voltage support zone - strategy 1

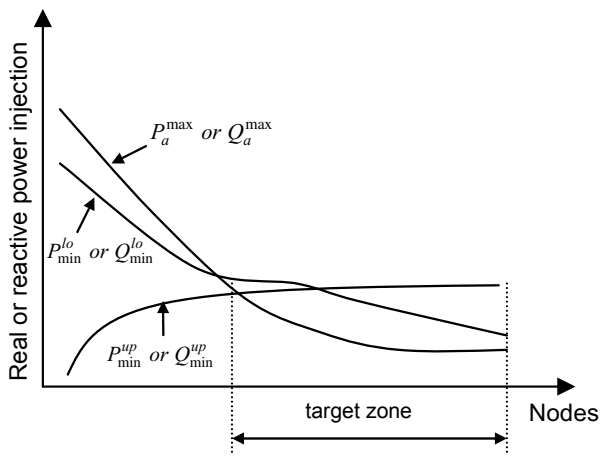

Fig. 2. Identification of voltage support zone - strategy 2

Applying (9) with different specified voltage $\Delta V_{a}$ at each node, the allowable incremental real power injections $\Delta P_{a}$ at a node can be calculated as:

$$
\left[\begin{array}{c}
\Delta P_{a(1 i)} \\
\vdots \\
\Delta P_{a(i i)} \\
\vdots \\
\Delta P_{a(n i)}
\end{array}\right]=\left[\begin{array}{c}
J_{R^{\prime}(1 i)}^{-1} \Delta V_{a(1)} \\
\vdots \\
J_{R^{\prime}(i i)}^{-1} \Delta V_{a(i)} \\
\vdots \\
J_{R^{\prime}(n i)}^{-1} \Delta V_{a(n)}
\end{array}\right]
$$

Thus $P_{a(i)}^{\max }$ can be obtained by:

$$
P_{a(i)}^{\max }=\min \left(\left[\begin{array}{c}
\Delta P_{a(1 i)} \\
\vdots \\
\Delta P_{a(i i)} \\
\vdots \\
\Delta P_{a(n i)}
\end{array}\right]\right)
$$

As $P_{\min }^{u p}, P_{\min }^{l o}$, and $P_{a}^{\max }$ have been numerically derived, the boundaries of the voltage support zones can be envisaged graphically shown in Fig. 1 and Fig. 2. Moreover, some of the common strategies can be adopted for the representative visualization.

Strategy 1: The boundary of the voltage support zone (from the downstream node) is a common point of intersection between $P_{m i n}^{u p}$ and $P_{m i n}^{l o}$ provided that $P_{m i n}^{u p}$ and $P_{m i n}^{l o}$ at the point of intersection are both lower than the $P_{a}^{\max }$.

Strategy 2: The boundary of the voltage support zone (from the 
downstream node) is a common point of intersection between $P_{\text {min }}^{u p}$ and $P_{a}^{\max }$ if either $P_{\text {min }}^{u p}$ or $P_{\min }^{l o}$ at the point of intersection is higher than the $P_{a}^{\max }$.

Since the voltage support zones for $P$ injections and $Q$ injections have been identified separately, the search space for optimal sizing and siting of DG units and shunt capacitors can be restricted to the corresponding identified zones.

As stated in the previous subsection, the linearised relationships of $P-V$ and $Q-V$ are only valid under the assumption of small incremental $\Delta P$ or $\Delta Q$ injections. The indices $P_{m i n}^{u p}$, $P_{\min }^{l o}$ and $P_{a}^{\max }$ are predicted based on these linearised relationships. In this paper, it is assumed that incremental $\Delta P$ and $\Delta Q$ injection will be made without applying any correction. However, it should be noted that the numerical values of these indices may not be accurate when an investigated radial distribution system is heavily loaded and large amounts of $P$ and $Q$ injections are needed to maintain system voltage. In this case, the correction of the predicted indices should be made in order to obtain the accurate values for $P_{\text {min }}^{u p}, P_{\text {min }}^{l o}$ and $P_{a}^{\max }$. The correction procedure of predicted $P_{\min }^{u p}$ is described here and the corrections for the remaining indices can be achieved by using the same approach. The correction of predicted $P_{m i n}^{u p}$ can be performed by adopting the following steps:

1) Obtain the initial predicted values of $P_{m i n}^{u p}$ without any incremental $\Delta P$ injection.

2) Apply the initial predicted $P_{\text {min }}^{u p}$ at a specified node with corresponding value and perform the power flow calculation.

3) Obtain the updated value of $P_{m i n}^{u p}$ and add the updated value to the old ones.

4) Perform Step 3 iteratively until the new $P_{\text {min }}^{u p}=0$ and record the final accumulated $P_{\text {min }}^{u p}$.

5) Remove the previous added $P$ injection and go to Step 2 to try remaining nodes until all nodes are evaluated.

\section{Proposed Approach}

Based on the derivation described in the Section II.A and Section II.B, the proposed approach for identification of voltage support zone for real power injection is summarised. The same approach can also be used for identification of voltage support zone for reactive power injection. The proposed approach consists of the following steps:

Step 1: Obtain the Jacobian matrix by performing NewtonRaphson power flow calculation and derive the linearised relationship matrix $J_{R^{\prime}}$ by using (1), (2), and (3).

Step 2: Calculate the desired voltage improvement $\Delta V_{d}$ and maximum allowable voltage improvement $\Delta V_{a}$ for each node by using (8) and (16) respectively.

Step 3: Form the desired real power injection matrix $P_{d}$ by applying (9) to each node.

Step 4: Build matrices $P_{d}^{u p}$ and $P_{d}^{l o}$ by using feeder topology matrices $M_{u p}$ and $M_{l o}$ and applying (11) and (12) respectively.

Step 5: Obtain minimum desired real power injection matrices $P_{\text {min }}^{u p}$ and $P_{\text {min }}^{l o}$ by obtaining the maximum values in each column of $P_{d}^{u p}$ and $P_{d}^{l o}$ as indicated in (13) and (14) respectively.

Step 6: Obtain the maximum allowable real power injection matrix $P_{a}^{\max }$ by applying (17) and (18) to each system node.
Step 7: In order to address the nonlinear P-V characteristic, the corrections of $P_{m i n}^{u p}, P_{m i n}^{l o}$ and $P_{a}^{\max }$ can be done by the proposed correction procedure. The identified voltage support zone for $P$ injection can be obtained by applying the proposed strategies.

\section{MAXIMUm Loss Reduction}

The total active power loss $(L)$ for a distribution network can be calculated as [5]:

$$
L=\sum_{j=1}^{N-1} I_{(j)}^{2} R_{(j)}
$$

where $I_{(j)}$ is the magnitude of current for feeder section $j$, $R_{(j)}$ is the resistance of feeder section $j$ and $N-1$ is the total number of feeder sections, for $N$ nodes.

The total active power loss is contributed by both active currents $\left(I_{p}\right)$ and reactive currents $\left(I_{q}\right)$. Therefore, (19) can be rewritten as [5]:

$$
L=\sum_{j=1}^{N-1} I_{p(j)}^{2} R_{(j)}+\sum_{j=1}^{N-1} I_{q(j)}^{2} R_{(j)}
$$

It is assumed that the incremental change in nodal voltages will not affect the magnitude of active and reactive current at each node. The new power loss due to active current injection $\left(I_{d g(k)}\right)$ at node $k$ can be calculated as:

$$
\begin{aligned}
L_{p}^{\text {new }}= & \sum_{j=1}^{N-1} I_{q(j)}^{2} R_{(j)}+\sum_{j=1}^{k-1}\left(I_{p(j)}-I_{d g(k)}\right)^{2} R_{(j)} \\
& +\sum_{j=k}^{N-1} I_{p(j)}^{2} R_{(j)}
\end{aligned}
$$

Subtracting (21) from (20), the change of system active loss $\Delta L_{p}$ due to real power injection $P_{d g(k)}$ at node $k$ can be obtained as:

$$
\begin{aligned}
\Delta L_{p(k)} & =2 I_{d g(k)} \sum_{j=1}^{k-1} I_{p(j)} R_{(j)}-I_{d g(k)}^{2} \sum_{j=1}^{k-1} R_{(j)} \\
& =\frac{2 P_{d g(k)}}{\left|V_{(k)}\right|} \sum_{j=1}^{k-1} I_{p(j)} R_{(j)}-\left(\frac{P_{d g(k)}}{\left|V_{(k)}\right|}\right)^{2} \sum_{j=1}^{k-1} R_{(j)}(22)
\end{aligned}
$$

The maximum loss reduction can be achieved if

$$
\frac{\partial L_{p(k)}}{\partial P_{d g(k)}}=0
$$

Therefore, the desired active power injection $\left(P_{o p t(k)}\right)$ for maximum loss reduction can be obtained as:

$$
P_{o p t(k)}=\left|V_{(k)}\right| \frac{\sum_{j=1}^{k-1} I_{p(j)} R_{(j)}}{\sum_{j=1}^{k-1} R_{(j)}}
$$


Similarly, the desired reactive power injection $\left(Q_{o p t(k)}\right)$ for maximum loss reduction can be obtained as:

$$
Q_{o p t(k)}=\left|V_{(k)}\right| \frac{\sum_{j=1}^{k-1} I_{q(j)} R_{(j)}}{\sum_{j=1}^{k-1} R_{(j)}}
$$

\section{Cost Optimisation For MaXimum Voltage SUPPORT}

The optimal sizes and locations of DG units and shunt capacitors are identified by minimising the total investment cost. The operation and maintenance $(\mathrm{O} \& \mathrm{M})$ costs are not considered in this formulation. The objective is to maintain system voltages within allowable limits by minimising the cost investment of DG units and shunt capacitors.

\section{A. Objective Function}

The objective function considered in this paper includes the investment cost for DG units $\left(C_{d g}\right)$ and the investment cost for shunt capacitors $\left(C_{c a p}\right)$. The total investment $\left(C_{t}\right)$ for maintaining the voltage can be calculated as:

$$
C_{t}\left(U_{d g}, U_{c a p}\right)=C_{d g}\left(U_{d g}\right)+C_{c a p}\left(U_{c a p}\right)
$$

where $U_{d g}$ and $U_{c a p}$ are two vectors indicating the sizes of DG units and shunt capacitors, respectively.

These two vectors can be expressed as:

$$
\begin{gathered}
U_{d g}=\left[U_{d g(1)}, \ldots, U_{d g(i)}, \ldots, U_{d g(N)}\right] \\
U_{c a p}=\left[U_{c a p(1)}, \ldots, U_{c a p(i)}, \ldots, U_{c a p(N)}\right]
\end{gathered}
$$

where $U_{d g(i)}$ is the size of the DG unit installed at node $i$, and $U_{c a p(i)}$ is the size of the shunt capacitor installed at node $i$.

The decision variables for building new DG units $\left(D_{d g}\right)$ and shunt capacitors $\left(D_{c a p}\right)$ can be obtained as:

$$
\begin{gathered}
D_{d g(i)}= \begin{cases}1 & \text { if } U_{d g(i)}>0 \\
0 & \text { otherwise }\end{cases} \\
D_{\text {cap }(i)}= \begin{cases}1 & \text { if } U_{c a p(i)}>0 \\
0 & \text { otherwise }\end{cases}
\end{gathered}
$$

1) Investment Cost for DG units: The total investment cost for DG units includes the installation cost and the cost for purchasing DG units. It is given by:

$$
C_{d g}\left(U_{d g}\right)=\sum_{i=1}^{N} D_{d g(i)}\left(\operatorname{Ins}_{(i)}^{d g}+r_{(i)}^{d g} U_{d g(i)}\right)
$$

where $\operatorname{Ins} s_{(i)}^{d g}$ is the cost for installation of DG unit at system node $i$ (in \$/installaion) and $r(i)^{d g}$ is the capacity cost, which is associated with the size of the DG unit (in $\$ / \mathrm{kVA}$ ).
2) Investment Cost for Shunt Capacitors: The total investment cost for shunt capacitors comprises of the installation cost and the investment cost for new shunt capacitors, which can be given as:

$$
C_{c a p}\left(U_{c a p}\right)=\sum_{i=1}^{N} D_{c a p(i)}\left(\operatorname{Ins}_{(i)}^{c a p}+r_{(i)}^{c a p} U_{c a p(i)}\right)
$$

where $\operatorname{Ins}_{(i)}^{c a p}$ is the cost for installing a new shunt capacitor at system node $i$ (in \$/installation) and $r_{(i)}^{c a p}$ is the cost associated with the size of the shunt capacitor (in $\$ / \mathrm{kVAr}$ ).

\section{B. System Constraints}

The inequality constraints in terms of nodal voltage limits and the feeder loading limits are included in the proposed optimisation problem. For different loading levels, the nodal voltages should be within the acceptable limits as indicated by (33). Similarly, the feeder loading should be less than the current rating of each feeder section as indicated by (34).

$$
\begin{gathered}
V_{l o} \leq V_{\text {node }(i)} \leq V_{u p} \\
\left|I_{f d r(j)}\right| \leq I_{\text {rating }}
\end{gathered}
$$

\section{Solution Algorithm}

The PSO search method is used in this paper to solve the formulated cost optimisation problem. The PSO search method is an intelligence-based heuristic optimisation technique [12], which finds optimal solution through the problem space by using specified number of particles. Unlike conventional analytical methods, PSO is a gradient-free search method which can be used to solve non-continuous and non-differentiable optimisation problems (such as MINLP). Although it could be difficult to guarantee the global optimal solution by using PSO, convincing and near optimal results can still be obtained with an appropriate representation of the optimisation problem.

The movement of each particle towards the optimal solution is based on its own inertia, experience and the good solutions provided by other particles. The new position of each particle is updated iteratively based on its position at previous time step and the velocity at current time step. The movement of the particle can be mathematically represented as [10]:

$$
x_{l}(t s)=x_{l}(t s-1)+v_{l}(t s)
$$

where $x_{l}(t s)$ is the new position of particle $l$ at time step $t s$ and $v_{l}(t s)$ is the velocity of particle $l$ at time step $t s$.

The velocity of each particle at each time step can be obtained as:

$$
\begin{aligned}
v_{l}(t s)= & v_{l}(\text { ts }-1)+c_{1} \cdot \operatorname{rand}_{1} \cdot\left(\text { pbest }_{l}-x_{l}(t s-1)\right) \\
& +c_{2} \cdot \operatorname{rand}_{2} \cdot\left(S_{\text {best }}-x_{l}(\text { ts }-1)\right)
\end{aligned}
$$

where $c_{1}$ and $c_{2}$ are two acceleration factors, rand $d_{1}$ and rand $_{2}$ are two random numbers, pbest ${ }_{l}$ is the best position of each particle in the whole search space, and $S_{\text {best }}$ is the best position found by all particles. 


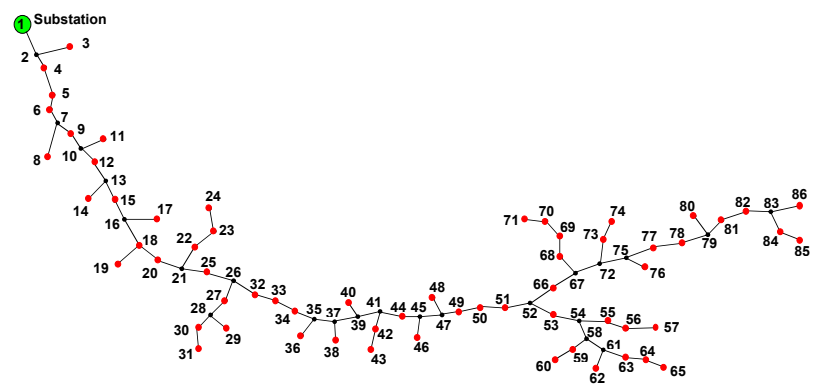

Fig. 3. 86 node test feeder

TABLE I

Cost Data Used in Simulations

\begin{tabular}{c||c}
\hline \hline DG Capacity Cost & $\$ 70 / \mathrm{kVA}$ \\
\hline DG Installation Cost & $\$ 160,000$ \\
\hline Shunt Capacitor Capacity Cost & $\$ 15 / \mathrm{kVAr}$ \\
\hline Shunt Capacitor Installation Cost & $\$ 15,000$ \\
\hline \hline
\end{tabular}

Generally, $c_{1}+c_{2}$ will be less than or equal to 4.0 to ensure the convergence of the optimisation [13]. In this paper, $c_{1}$ and $c_{2}$ are set to be 2 and 2 respectively. A penalty function (in terms of an additional penalty term $\left.C_{p}\left(V_{\text {node }}, I_{f d r}\right)\right)$ will be used in case of a constraint violation. If the system constraints do not get violated, then the penalty term will be zero. Thus, the modified objective function can be rewritten as:

$$
\begin{aligned}
C_{t}\left(U_{d g}, U_{c a p}\right)= & C_{d g}\left(U_{d g}\right)+C_{c a p}\left(U_{c a p}\right) \\
& +C_{p}\left(V_{\text {node }}, I_{f d r}\right)
\end{aligned}
$$

\section{Simulation Results}

The sample system under study is a rural $11 \mathrm{kV}$ radial distribution feeder from New South Wales, Australia. The topology of this feeder is shown in Fig. 3. The substation voltage of this feeder is maintained constant at $0.9732 \mathrm{pu}$. The impedance of the main feeder sections is $0.315+\mathrm{j} 0.354$ $\Omega / \mathrm{km}$. It has been observed that the peak real and reactive power demands for the feeder are $1931 \mathrm{~kW}$ and $935 \mathrm{kVAr}$ respectively at 7:00am on 14/12/2007. It is assumed that this peak demand is a $100 \%$ loading condition for the feeder. The associated cost data given in Table I for this case study has been obtained from the respective distribution utility. In this section, the voltage support zones are identified and the cost optimisation is done for different loading levels. The minimum $P$ or $Q$ injection for maximising the voltage support is evaluated and the related loss reduction will be analysed. The search for optimal sizing and siting of DG units and shunt capacitors will be carried out with the consideration of the identified voltage support zones.

\section{A. Voltage Support Zones for the Test Feeder}

It is assumed that the acceptable voltage limits are between $0.95 \mathrm{pu}$ to $0.9732 \mathrm{pu}$ (substation voltage). The voltage support zones for independent $P$ and $Q$ injection at peak load condition are shown in Figs. 4 and 5 respectively. It can be seen that
TABLE II

VOLTAGE Support ZONES FOR REAL POWER INJECTION

\begin{tabular}{c||c|c|c}
\hline \multicolumn{1}{c||}{\multirow{2}{*}{$\begin{array}{c}\text { Loading } \\
\text { Levels }\end{array}$}} & \multicolumn{3}{c}{ Node Numbers for the Identified Zone } \\
\cline { 2 - 4 } & $V_{\text {low }}=0.95 \mathrm{pu}$ & $V_{\text {low }}=0.96 \mathrm{pu}$ & $V_{\text {low }}=0.97 \mathrm{pu}$ \\
\hline \hline $100 \%$ & $52-86$ & $49-86$ & $32-86$ \\
\hline $90 \%$ & $52-86$ & $50-86$ & $33-86$ \\
\hline $80 \%$ & $52-86$ & $50-86$ & $34-86$ \\
\hline $70 \%$ & $52-86$ & $51-86$ & $37-86$ \\
\hline $60 \%$ & $53-65$ & $52-86$ & $39-86$ \\
\hline $50 \%$ & $53-65$ & $52-86$ & $46-86$ \\
\hline \hline
\end{tabular}

TABLE III

VOLTAGE SUPPoRt ZONES FOR REACTIVE POWER INJECTION

\begin{tabular}{c||c|c|c}
\hline \hline \multirow{2}{*}{\multicolumn{1}{c||}{$\begin{array}{c}\text { Loading } \\
\text { Levels }\end{array}$}} & \multicolumn{3}{c}{ Node Numbers for the Identified Zone } \\
\cline { 2 - 4 } & $V_{\text {low }}=0.95 \mathrm{pu}$ & $V_{\text {low }}=0.96 \mathrm{pu}$ & $V_{\text {low }}=0.97 \mathrm{pu}$ \\
\hline \hline $100 \%$ & $52-86$ & $49-86$ & $32-86$ \\
\hline $90 \%$ & $52-86$ & $50-86$ & $32-86$ \\
\hline $80 \%$ & $52-86$ & $50-86$ & $32-86$ \\
\hline $70 \%$ & $52-86$ & $51-86$ & $35-86$ \\
\hline $60 \%$ & $53-65$ & $52-86$ & $39-86$ \\
\hline $50 \%$ & $53-65$ & $52-86$ & $44-86$ \\
\hline \hline
\end{tabular}

the boundaries of the voltage support zones in this case can be identified by using the Strategy 1 as discussed in Section II. The other voltage support zones for different loading levels and allowable voltage limits are summarised in Table II and Table III. It can be observed that the voltage support zones expand with the increase in loading level and also when the lower limit of the acceptable voltage is increased. It can also be seen that the voltage support zones will be much larger in case of the lower voltage limit of $0.97 \mathrm{pu}$ than that of $0.95 \mathrm{pu}$ and $0.96 \mathrm{pu}$.

\section{B. Voltage Support Vs. Maximum Loss Reduction}

The comparison of desired real and reactive power injections for voltage support and loss minimisation (for $100 \%$ loading condition and 50\% loading condition) are shown in Fig. 6 to Fig. 9 respectively.

It can be seen in Fig. 6 that the amount of $P$ injection for voltage support $\left(P_{\text {min }}\right)$ is almost the same as the optimal amount of $P$ injection $\left(P_{o p t}\right)$ for maximum loss reduction if $P$ injection is located between node 41 and node 86 . In this case, the voltage support can be ensured by minimising the system loss. But, for $Q$ injection shown in Fig. 7, the desired $Q$ injection for voltage support is higher than the optimal $Q$ for loss reduction. Moreover, if the shunt capacitor is placed close to the end of the feeder, the system loss will be higher than the system loss $\left(L_{o r}\right)$ without shunt capacitor. For 50\% loading condition, it can be seen in Fig. 8 and Fig. 9 that the real and reactive power injections are lower than the optimal real and reactive power injections for maximum loss reduction.

\section{Results for Cost Optimisation}

It is assumed that the maximum allowable $P$ and $Q$ penetration is $50 \%$ and the acceptable lower voltage limit is 0.95 


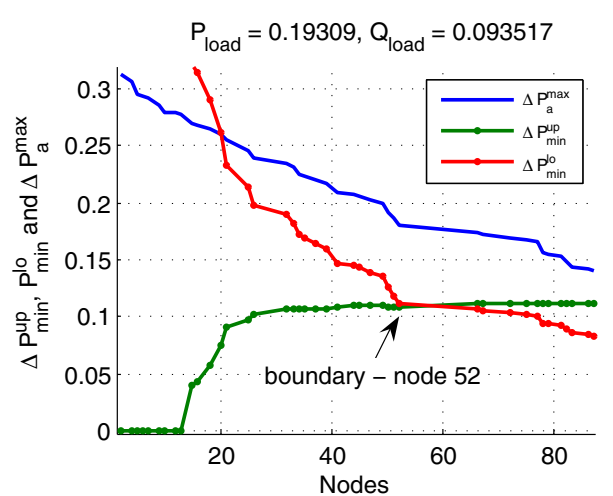

Fig. 4. Voltage support zone for $\mathrm{P}$ injection (100\% loading condition, $V_{\text {low }}$ $=0.95$ )

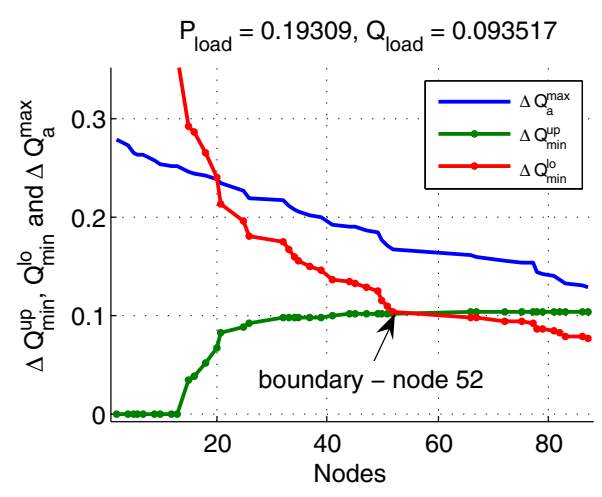

Fig. 5. Voltage support zone for Q injection (100\% loading condition, $V_{l o w}$ $=0.95)$

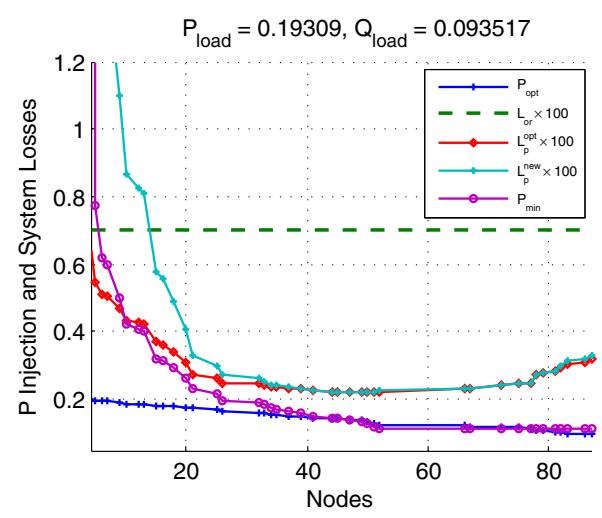

Fig. 6. Voltage support and loss minimisation with P injection (100\% loading condition, $V_{\text {low }}=0.95$ )

pu. The real power injection is assumed to be from a diesel engine driven synchronous generator operated with unit power factor. Fig. 10 shows the voltage improvement at peak loading condition when the DG unit and shunt capacitor are placed at the optimal locations with optimal sizes. The simulation results for six loading levels are shown in Table IV. It can be seen that for light loading condition (50\% of the peak loading), only one shunt capacitor is required at node 54 for requisite voltage support. The total cost investment is $\$ 17,281$. As the load increases, the voltage support merely by using $50 \% Q$ penetration is not sufficient. Therefore, real power injection

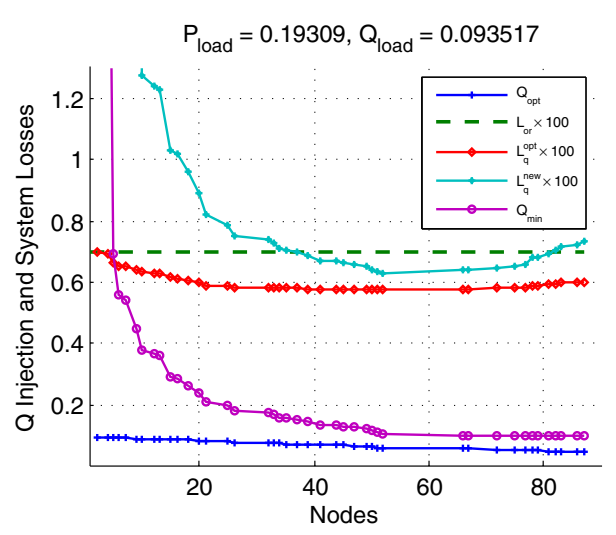

Fig. 7. Voltage support and loss minimisation with Q injection (100\% loading condition, $V_{\text {low }}=0.95$ )

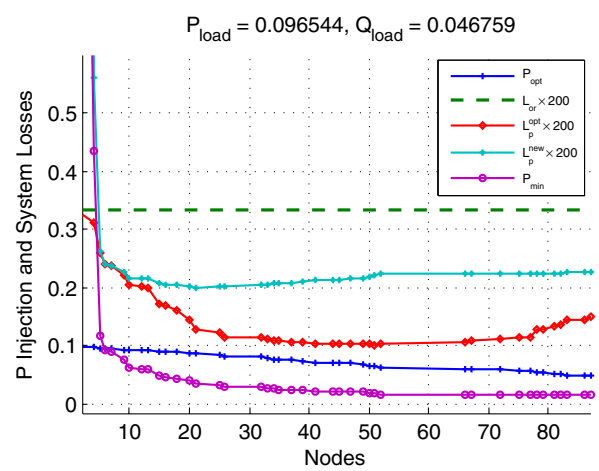

Fig. 8. Voltage support and loss minimisation with P injection (50\% loading condition, $V_{\text {low }}=0.95$ )

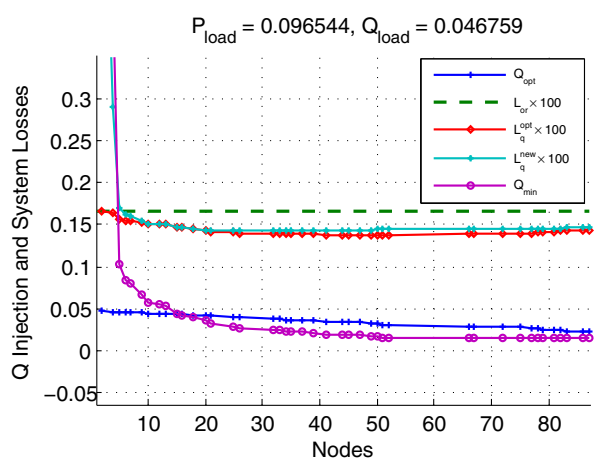

Fig. 9. Voltage support and loss minimisation with Q injection (50\% loading condition, $V_{\text {low }}=0.95$ )

by means of DG unit will be demanded in parallel with the shunt capacitor to maintain the system voltage. Thus, it can be observed that for the loading condition above $60 \%$, there will be one DG unit and one shunt capacitor in the system at various specified nodes with different cost investment.

\section{Conclusions}

An analytical approach has been proposed for identifying the voltage support zones with minimum real and reactive power injections. This formulation has been applied in relation to a realistic radial distribution feeder ensuring acceptable 
TABLE IV

SIMULATION RESULTS FOR COST OPTIMISATION

\begin{tabular}{|c|c|c|c|c|}
\hline $\begin{array}{c}\text { Loading } \\
\text { Levels }\end{array}$ & Device & Location & Size & $\begin{array}{c}\text { Total } \\
\text { Investment }\end{array}$ \\
\hline \multirow{2}{*}{$100 \%$} & DG unit & 75 & $623 \mathrm{~kW}$ & \multirow{2}{*}{$\$ 225,559$} \\
\hline & $\begin{array}{c}\text { Shunt } \\
\text { Capacitor }\end{array}$ & 59 & $465 \mathrm{kVAr}$ & \\
\hline \multirow[t]{2}{*}{$90 \%$} & DG unit & 59 & $476 \mathrm{~kW}$ & \multirow[t]{2}{*}{$\$ 214,617$} \\
\hline & $\begin{array}{c}\text { Shunt } \\
\text { Capacitor }\end{array}$ & 72 & $420 \mathrm{kVAr}$ & \\
\hline \multirow[t]{2}{*}{$80 \%$} & DG unit & 59 & $335 \mathrm{~kW}$ & \multirow[t]{2}{*}{$\$ 203,991$} \\
\hline & $\begin{array}{c}\text { Shunt } \\
\text { Capacitor }\end{array}$ & 86 & $371 \mathrm{kVAr}$ & \\
\hline \multirow[t]{2}{*}{$70 \%$} & DG unit & 62 & $184 \mathrm{~kW}$ & \multirow[t]{2}{*}{$\$ 192,805$} \\
\hline & $\begin{array}{c}\text { Shunt } \\
\text { Capacitor }\end{array}$ & 75 & $327 \mathrm{kVAr}$ & \\
\hline \multirow[t]{2}{*}{$60 \%$} & DG unit & 53 & $61 \mathrm{~kW}$ & \multirow[t]{2}{*}{$\$ 183,448$} \\
\hline & $\begin{array}{c}\text { Shunt } \\
\text { Capacitor }\end{array}$ & 53 & $280 \mathrm{kVAr}$ & \\
\hline $50 \%$ & $\begin{array}{c}\text { Shunt } \\
\text { Capacitor }\end{array}$ & 54 & $152 \mathrm{kVAr}$ & $\$ 17,281$ \\
\hline
\end{tabular}

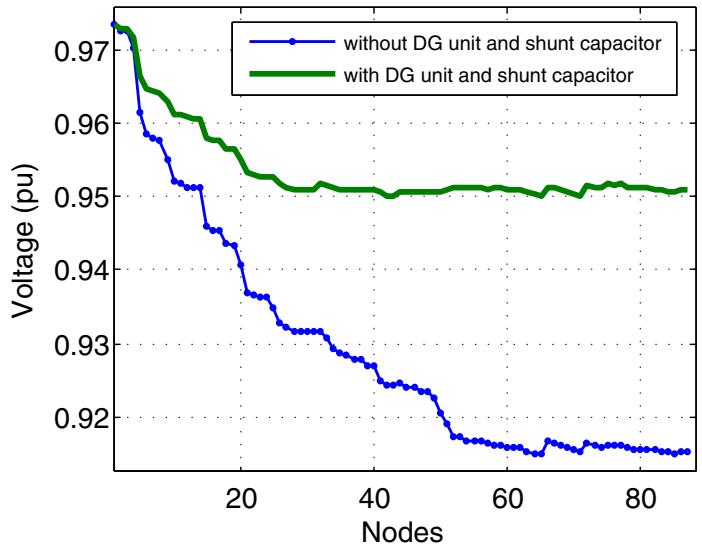

Fig. 10. System voltage profiles at $100 \%$ loading condition

voltage at each node. It has been realised that the real and reactive power injections in the target zones with specific nodes guarantee requisite voltage improvement. The comparison of active and reactive power injections for maximum voltage support and maximum loss reduction has been graphically presented for peak load as well as light load condition. PSO based heuristic search method is demonstrated for sizing and siting of DG units and shunt capacitors with minimum cost investment. The results are highlighted for different loading conditions.

\section{ACKNOWLEDGMENT}

Authors would like to thank Integral Energy personnel for providing data and information on the network.

\section{REFERENCES}

[1] J. A. Pecas Lopes, N. Hatziargyriou, J. Mutale, P. Djapic, N. Jenkins, "Integrating Distributed Generation into Electric Power Systems: A Review of Drivers, Challenges and Opportunities," Electric Power Systems Research, Vol. 77, pp. 1189-1203, July 2007.
[2] R. A. Gallego, A. Monticelli, R. Romero, "Optimal Capacitor Placement in Radial Distribution Networks," IEEE Trans. Power Systems, Vol. 16, pp. 630-637, November 2001.

[3] J. J. Grainger, S. H. Lee, "Optimum Size and Location of Shunt Capacitors for Reduction of Losses On Distribution Feeders," IEEE Trans. Power Apparatus and Systems, Vol. PAS-100, pp. 1105-1118, March 1981.

[4] I. C. da Silva, S. Carneiro, E. J. de Oliveira, J. de Souza Costa, J. L. R. Pereira, P. A. N. Garcia, "A Heuristic Constructive Algorithm for Capacitor Placement on Distribution Systems," IEEE Trans. Power Systems, Vol. 23, pp.1619-1626, November 2008.

[5] S. F. Mekhamer, M. E. El-Hawary, S. A. Soliman, M. A. Moustafa, M. M. Mansour, "New Heuristic Strategies for Reactive Power Compensation of Radial Distribution Feeders," IEEE Trans. Power Delivery, Vol. 17, pp. 1128-1135, October 2002.

[6] M. Chis, M. M. A. Salama, S. Jayaram, "Capacitor Placement in Distribution Systems Using Heuristic Search Strategies," Generation, Transmission and Distribution, IEE Proceedings , Vol. 144, pp.225-230, May 1997.

[7] E. G. Carrano, R. T. N. Cardoso, R. H. C. Takahashi, C. M. Fonseca, O. M. Neto, "Power Distribution Network Expansion Scheduling using Dynammic Programming Genetic Algorithm," IET, Generation, Transmission and Distribution, Vol. 2, pp. 444-455, May 2008.

[8] A. Ahuja, S. Das, A. Pahwa, "An AIS-ACO Hybrid Approach for MultiObjective Distribution System Reconfiguration," IEEE Trans. Power Systems, Vol. 22, pp. 1101-1111, August 2007.

[9] J. M. Nahman, D. M. Peric, "Optimal Planning of Radial Distribution Networks by Simulated Annealing Technique," IEEE Trans. Power Systems, Vol. 23, pp. 790-795, May 2008.

[10] Y. del Valle, G. K. Venayagamoorthy, S. Mohagheghi, J.-C. Hernandez, R. G. Harley, "Particle Swarm Optimisation: Basic Concepts, Variants and Applications in Power Systems," IEEE Trans. Evolutionary Computation, Vol. 12, pp. 171-195, April 2008.

[11] P. Kundur, Power System Stability and Control. USA: McGraw-Hill, 1994, p. 1176.

[12] J. Kennedy, R. Eberhart, "Particle Swarm Optimisation," in Proc. 6th Int. Symp. Micro Machine and Human Science (MHS), October 1995, pp. $39-43$.

[13] R. Eberhart, Y. Shi, J. Kennedy, Swarm Intelligence. San Diego: Morgan Kaufmann, 2001, p. 512.

Kai Zou received the B.Eng. degree in electrical power engineering in 2005 from the Huazhong University of Science and Technology, China, and the M.Eng. degree in 2006 from the University of Wollongong, Australia. He is currently working towards his Ph.D. degree in the area of power distribution system planning and optimisation with embedded generation at the University of Wollongong.

A. P. Agalgaonkar received the Ph.D. degree from Indian Institute of Technology-Bombay, Mumbai, India in 2006. Currently, he is working as a Postdoctoral Research Fellow at the School of Electrical, Computer, Telecommunications Engineering, University of Wollongong, Wollongong, Australia. He was associated with the School of Engineering, University of Tasmania as a Postdoctoral Research Fellow from October 2007 to January 2008. He also worked as a Scientist in the Energy Technology Centre, NTPC Limited, India from 2005 to 2007. His research interests include load modeling, impact of distributed generation on distribution systems, micro-grids, and condition monitoring of electrical equipments.

K. M. Muttaqi (M'01, SM'05) received the Ph.D. degree from Multimedia University, Malaysia, in 2001. Currently, he is an Associate Professor at the School of Electrical, Computer, and Telecommunications Engineering, University of Wollongong, Wollongong, Australia. He was associated with the University of Tasmania, Australia as a Research Fellow/Lecturer/Senior Lecturer from 2002 to 2007, and with the Queensland University of Technology, Australia as a Research Fellow from 2000 to 2002. Previously, he also worked for Multimedia University as a Lecturer for three years. His special fields of interests include distributed generation, renewable energy, distribution system automation, and power system planning.

S. Perera (M'95) received the B.Eng. degree in electrical power engineering from the University of Moratuwa, Sri Lanka, the M.Eng. degree from the University of New South Wales, Sydney, Australia, and the Ph.D. degree from the University of Wollongong, Wollongong, Australia. He had been a lecturer at the University of Moratuwa. Currently he is an Associate Professor with the University of Wollongong, where he is also the Technical Director of the Integral Energy Power Quality and Reliability Centre. 